\title{
Passer les frontières dans la Corne de l'Afrique : trois logiques de survie autour des figures du réfugié, du passeur et du rebelle
}

\section{Alexandre Lauret}

\section{(2) OpenEdition}

Journals

Édition électronique

URL : https://journals.openedition.org/echogeo/20232

DOI : $10.4000 /$ echogeo.20232

ISSN : 1963-1197

Éditeur

Pôle de recherche pour l'organisation et la diffusion de l'information géographique (CNRS UMR 8586)

Référence électronique

Alexandre Lauret, " Passer les frontières dans la Corne de l'Afrique : trois logiques de survie autour des figures du réfugié, du passeur et du rebelle », EchoGéo [En ligne], Sur le Vif, mis en ligne le 04 janvier 2021, consulté le 24 août 2021. URL : http://journals.openedition.org/echogeo/20232 ; DOI : https:// doi.org/10.4000/echogeo.20232

Ce document a été généré automatiquement le 24 août 2021.

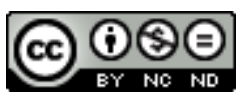

EchoGéo est mis à disposition selon les termes de la licence Creative Commons Attribution - Pas d'Utilisation Commerciale - Pas de Modification 4.0 International 


\title{
Passer les frontières dans la Corne de l'Afrique : trois logiques de survie autour des figures du réfugié, $d u$ passeur et du rebelle
}

\author{
Alexandre Lauret
}

1 À l'automne 2020, la guerre au Yémen est entrée dans sa sixième année depuis la prise de Sanaa par les rebelles Houthis en septembre 2014. Les affrontements entre rebelles, gouvernementaux, indépendantistes, islamistes radicaux et les alliés de la coalition arabe continuent de provoquer l'exil d'une partie de la population yéménite vers les États frontaliers comme Djibouti. Les conséquences sécuritaires et la possible déstabilisation régionale que représente la guerre du Yémen rappellent que les États de la Corne de l'Afrique et du Yémen ne sont pas avares de crises politiques et d'espaces fracturés. Cette région du sud de la mer Rouge est marquée par une forte instabilité des États. On peut brièvement citer l'instabilité de la Somalie depuis la décennie 1990, la fermeture des frontières entre l'Érythrée et Djibouti depuis 2008, enfin, les derniers troubles entre l'État central éthiopien et la région du Tigrée en 2020. La région a en outre connu deux sécessions étatiques au cours des dernières décennies (l'Érythrée en 1993 et le Somaliland en 1991) et pourrait en connaitre une troisième avec le Yémen du Sud. Les régimes autoritaires côtoient les États faillis ou en crise. La région est en proie à un fort morcellement territorial et politique, illustré, souvent, par une absence de dialogue entre les États et de fréquentes fermetures de frontières.

Ces crises ponctuent l'histoire des relations entre les États de la Corne de l'Afrique et du Yémen. La région représente un "centre endogène de haute pression" géopolitique (Gascon, 1990). Les crises y sont alors un fait marquant de la régulation sociale, politique et territoriale des États. Leur répétition dans un temps court - celle de la deuxième moitié du XXe siècle - donne à penser la crise comme un facteur structurel de l'évolution des États, des territoires et des formes de résistances. Pour autant, faut-il voir dans ces crises des obstacles aux échanges entre les populations habitant l'espace 
régional? Cet article souhaite montrer comment ces crises, loin de rompre les relations, favorisent l'apparition de nouveaux espaces d'échange entre populations de la Corne de l'Afrique et du Yémen.

3 L'hypothèse retenue ici est que les crises produisent des logiques de survie, voulues ou forcées, pour de nombreuses populations. Le terme de survie se base sur la notion de vie développée dans la pensée vitaliste de Georges Canguilhem : la survie est considérée comme une activité créatrice et innovatrice vis-à-vis de l'environnement dans lequel un être vivant se situe. Par conséquent, sera identifiée comme logique de survie toute dynamique productrice entre un être vivant et son environnement par laquelle, le premier tente d'assurer sa propre persistance et le dernier subit des altérations plus ou moins spontanées ou durables (Canguilhem, 2000 [1952]). Les crises produisent ainsi de nouveaux acteurs : migrants, réfugiés, contrebandiers, passeurs, rebelles etc. C'est par ces acteurs franchissant les frontières que l'on peut observer l'ouverture de nouveaux espaces d'échanges. Les frontières représentent autant la limite de la souveraineté d'un État qu'un lieu d'innovation socio-économique pour les populations riveraines (Bennafla et Peraldi, 2008). Dans la Corne de l'Afrique, les frontières prennent une symbolique particulière. Les logiques de peuplement ethnique ou clanique de part et d'autre des frontières redéfinissent les rapports entre populations et limites administratives. Des espaces linguistiques ou claniques homogènes favorisent l'amenuisement partiel de la frontière produisant une multitude de phénomènes sociaux ou politiques indépendamment des États (Feyissa et Hoehne, 2010).

4 La situation semble alors paradoxale. Les crises qui parcourent les États de la Corne de l'Afrique peuvent entraîner la fermeture des frontières nationales, mais cette fermeture permet la création d'espace opportun à l'innovation sociale: son franchissement traduit des logiques de survie donnant lieu à des nouvelles relations et pratiques entre l'acteur et l'espace, son milieu. Ainsi, les frontières érythréennes deviennent poreuses par leurs fermetures (Bozzini, 2014). C'est donc autour du passage des frontières que ces nouveaux espaces d'échanges peuvent apparaitre.

5 Cet article s'appuie sur trois figures sociales associées aux crises : le réfugié, le passeur et le rebelle. Chacune de ces figures nait de la crise et du franchissement de la frontière. Le réfugié est le revers d'une crise. Il est produit par une insécurité ponctuelle ou constante. Le passeur nait des circonstances d'une crise : la fermeture des frontières et la nécessité d'une population de fuir pour survivre. Enfin, le rebelle est un acteur direct de cette même crise. La naissance d'une rébellion et son maintien spatio-temporel peuvent être à l'origine d'une crise. Bien que ces trois figures soient ici érigées en tant que modèle pour cette démonstration, il faut rappeler que pour chacune, elles sont composées d'une grande diversité de cas et de situations sociales et politiques hétérogènes.

Chacune de ces figures a développé ses propres stratégies en lien avec le franchissement de la frontière à Djibouti. Elles permettent donc de réfléchir aux interactions entre les acteurs d'une crise et à la mobilisation de ressources permises par le franchissement de la frontière. Avant de les aborder, l'article présente la méthodologie de recherche appliquée à ces trois figures. La première est celle du réfugié yéménite en exil à Djibouti. Celui-ci présente une première logique de passage des frontières, celle de l'exode. L'exil donne l'occasion à une partie des réfugiés de réactualiser des liens historiques et familiaux sur plusieurs générations leur permettant une meilleure adaptation. La figure du passeur djiboutien faisant transiter 
des migrants éthiopiens représente une deuxième logique transfrontalière : celle des opportunités économiques naissant des crises et du passage de frontière. Le franchissement de la frontière offre aux passeurs et aux migrants l'accès à une forme de mobilité sociale. Enfin, la figure du rebelle afar de la guerre civile djiboutienne illustre une troisième logique : le franchissement de la frontière permet de continuer la lutte.

\section{Une recherche empirique}

7 Cet article évoque trois figures liées au passage des frontières dans la Corne de l'Afrique, qui renvoient à trois contextes différents. Les données récoltées illustrent le passage de la frontière, les conséquences de ces passages et les raisons qui ont amené à la franchir pour chaque acteur. Bien que ces figures soient différentes, la méthodologie de recherche est similaire. Les données primaires ont été récoltées majoritairement par des entretiens menés à Djibouti, dans le Nord de Djibouti Ville ou auprès de populations qui en étaient originaires.

8 Ces trois figures sont liées par des conditions socio-économiques précaires : le réfugié, le passeur et le rebelle sont des populations évoluant à la marge de leur État respectif. Les conditions liées à ces situations de marginalité sont autant sociales, politiques que géographiques : le passeur afar djiboutien est un jeune sans avenir car il vit dans une marge spatiale et politique. Le réfugié est contraint à l'exil par une situation politique qui se dégrade et par la perte de ses ressources socio-économiques et politiques. Enfin, le rebelle est doublement lié à la marge : la situation de marge est souvent cause de son engagement, autant que l'espace dans lequel la rébellion peut prospérer.

9 Les données sur les réfugiés yéménites ont été obtenues à Djibouti Ville ou dans le camp de réfugiés Markazi, à Obock. Ce dernier accueillait près de 1200 réfugiés yéménites en 2019. Ces données ont été récoltées en 2016 puis en 2018 et 2019, via des entretiens semi-directifs avec les réfugiés. Ils ont été rendus possibles par une coopération avec les travailleurs du Haut-Commissariat aux Réfugiés (HCR) et de l'Organisation Nationale pour les Assistés, les Réfugiés et les Sinistrés (ONARS Gouvernement djiboutien) à l'échelle locale, à Obock. Une centaine de réfugiés yéménites a été interrogée sur leur parcours de vie : les raisons et les routes de l'exil, puis l'installation à Djibouti. Les réfugiés sont arrivés en majorité en 2015 et en 2016. Très peu ont intégré le camp de réfugiés en 2018 ou 2019. Les matériaux sont donc extraits de récits vécus et narrés par les réfugiés eux-mêmes.

10 Les données sur les passeurs djiboutiens de Tadjoura ou d'Obock s'inscrivent dans le cadre d'une recherche doctorale commencée en 2017. Elles ont été obtenues par des entretiens ouverts ou semi-directifs. L'intégration aux réseaux des passeurs s'est faite de manière officieuse, en marge des institutions et des pouvoirs publics. Elle a été permise par des intermédiaires, à Djibouti ou en France. Nous avons ainsi pu observer, directement, les convois de migrants que les passeurs faisaient transiter, de nuit, sur le territoire djiboutien. Cette figure du passeur est donc construite par les données empiriques issues d'entretiens, de récits et d'observations sur le terrain.

11 Enfin, la figure du rebelle afar a été la plus difficile à construire. Trois raisons expliquent cette difficulté. Il y a d'abord une barrière temporelle. La guerre civile djiboutienne a eu lieu entre 1991 et 1994. Depuis, de nombreux anciens rebelles sont décédés ou n'ont pas souhaité revenir sur ces événements. La deuxième difficulté 
renvoie à une barrière géographique. Il existe toujours des rebelles afars (djiboutiens, érythréens ou éthiopiens) vivant dans les marges géographiques de l'extrême nord du territoire djiboutien. Il a été impossible de se rendre dans ces régions reculées, à l'accès formellement interdit pour tout étranger. La difficulté politique n'est pas la moindre : le rebelle afar représente une figure ennemie de l'État djiboutien. Évoquer la rébellion afar contre laquelle le régime, autoritaire, tente de lutter depuis 1991, est un sujet tabou à Djibouti. S'entretenir avec des rebelles afars expose à des sanctions, dont celle de l'expulsion de Djibouti (et donc du terrain de recherche). Les données ont ainsi été récoltées auprès d'anciens combattants à Djibouti ou auprès de partisans de la rébellion qui sont réfugiés politiques en France. Il s'agit donc de données datées (souvenirs de de la guerre) ou de la vision d'une lutte politico-armée depuis l'étranger, en Europe.

\section{La figure du réfugié yéménite ou la logique de l'exil au- delà de la frontière}

12 La première figure transfrontalière étudiée est celle du réfugié yéménite. Le réfugié est autant un acteur de la crise que son revers. C'est parce que la crise (ici la guerre) dégénère qu'une population décide de tout quitter pour survivre. Le réfugié est un exemple de cas permettant de penser aux articulations qui se créent entre exil et revalorisation de la mémoire familiale comme ressources à l'adaptation dans une nouvelle société. Cette partie s'ouvre sur la contextualisation de la guerre civile et sur les parcours d'exil des réfugiés. Le détour par les communautés yéménites historiques d'Érythrée et de Djibouti du début du XXe siècle montre que les parcours d'exode actuel s'inscrivent dans la continuité d'une histoire pluri-générationnelle. Enfin, en arrivant à Djibouti, les réfugiés yéménites réactivent les réseaux de connaissances hérités de leurs histoires personnelles.

13 La guerre civile yéménite qui débute en 2014 entraîne l'exil de plus de 190000 Yéménites au-delà des frontières ${ }^{1}$. Ce conflit s'inscrit dans la continuité d'une longue instabilité qui dure depuis l'unification du pays en $1990^{2}$ (Mermier, 2018). En septembre 2014, les rebelles Houthis prennent le contrôle de la capitale, Sanaa. C'est le début de leur conquête territoriale : ils prennent la côte ouest du pays, bordant la mer Rouge. Puis, ils tentent de prendre la ville portuaire d'Aden, au sud, au printemps 2015. L'avancée des Houthis, du Nord vers le Sud, se fait en parallèle d'un flux de déplacés, puis de réfugiés, qui traversent le détroit de Bab el Mandeb. Ces réfugiés trouvent refuge, pour une partie d'entre eux, à Djibouti, où les Nations unies et le gouvernement djiboutien ont mis en place le camp de réfugiés de Markazi, à Obock, au Nord du pays.

Selon leur origine géographique, les réfugiés empruntent deux routes migratoires pour quitter le Yémen. La première, à l'Ouest, draine des populations de villes du Nord (Hodeïda ou Sanaa), qui descendent le long de la côte de la Mer Rouge jusqu'aux villes côtières du Sud (Mocha, Dhubab, Bab el Mandeb). Ces populations du Nord rejoignent alors celles de ces villes côtières qui tentent de quitter le Yémen. La seconde route migratoire est centrée sur les régions de Taïz et d'Aden. Leurs habitants se dirigent vers les quartiers portuaires d'Aden ou les espaces côtiers périphériques de la ville afin de trouver une embarcation pour traverser le golfe d'Aden en direction de Djibouti.

Le passage de la frontière se matérialise par la traversée de la mer par le détroit de Bab el Mandeb ou les eaux du golfe d'Aden. La frontière politique est ici naturelle: des terres séparées par une étendue d'eau. Les Yéménites arrivés dans les villes portuaires 
de la côte sud-est du Yémen cherchent donc à négocier leurs places sur des embarcations diverses: embarcation de pêcheurs, boutres de commerce, tankers ou porte-conteneurs quittant la ville.

Le réfugié apparaît au moment où le Yéménite franchit la mer et atteint la côte djiboutienne. Le franchissement de la frontière équivaut à un glissement de condition, marqué par le passage du statut de citoyen à celui de réfugié3. Si la traversée de la mer permet la survie du corps et de la mémoire, c'est aussi une perte violente de tous les symboles vivants de l'identité d'une personne (Adnan, 2020). L'exil est un tout: géographique, économique et identitaire. Mais les réfugiés ne sont pas uniquement porteurs des malheurs et des souffrances qu'ont entrainés la guerre civile yéménite et leur exil. Leur pérennité passe aussi par la mémoire. Ils sont porteurs, chacun, de leur histoire personnelle et familiale, notamment celle de leurs parents ou grands-parents. Ils véhiculent ainsi une double mémoire: celle de l'exil d'un peuple et celle, plus individuelle, de trajectoires historiques familiales.

Illustration 1 - Parcours migratoires des réfugiés et passeurs

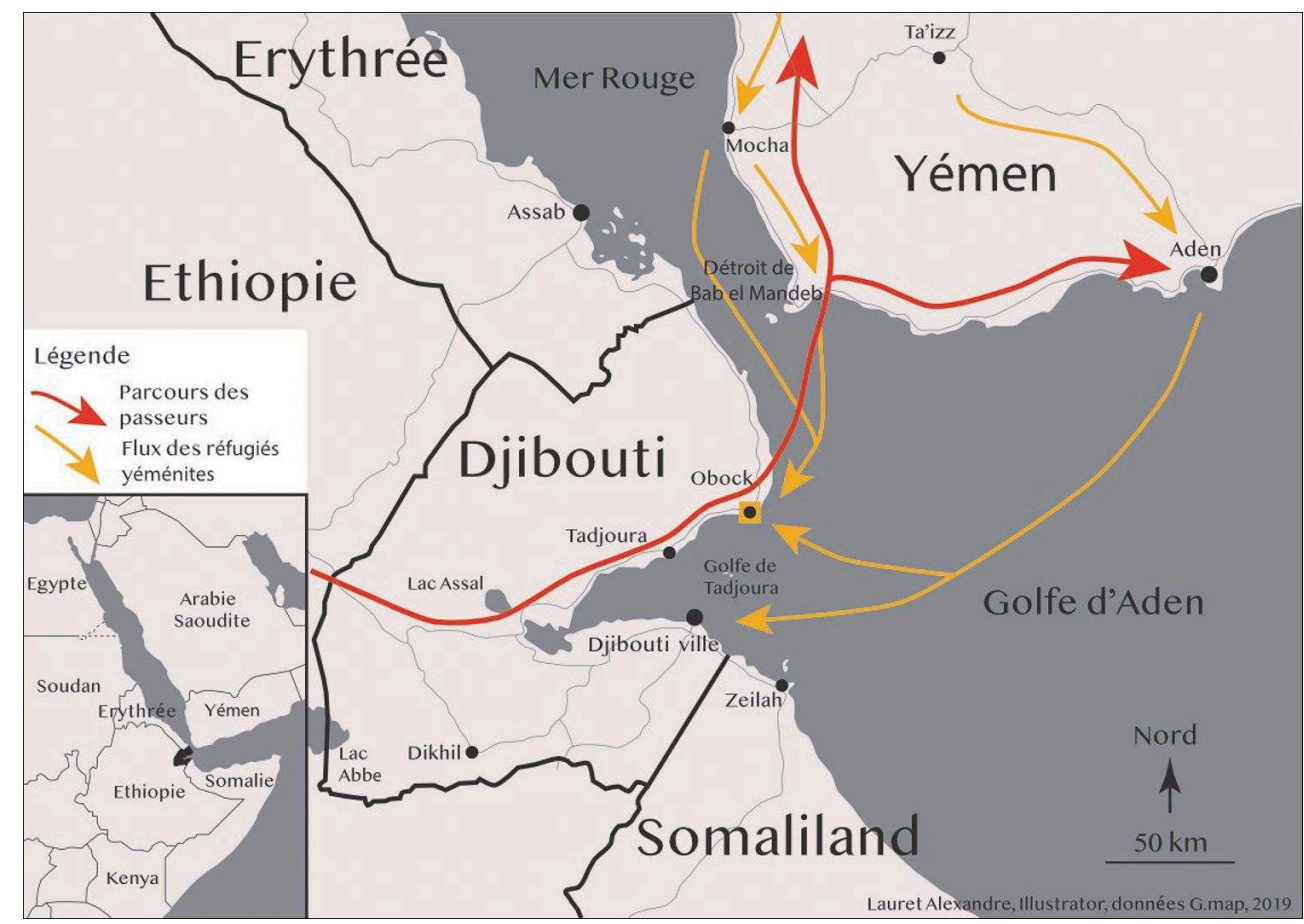

Données G map, 2019. Auteur : A. Lauret

17 Fuir la guerre civile yéménite à Djibouti représente une première stratégie de survie, à travers l'exil. Il s'agit de sauver sa vie et celle de sa famille face au danger du conflit. Une deuxième logique de survie se manifeste ensuite chez certains réfugiés yéménites. En arrivant à Djibouti, ils réactualisent leur capital social, à travers des réseaux d'entraides hérités de leurs mémoires familiales.

L'exil à Djibouti peut être considéré comme la dernière vague migratoire des populations yéménites autour du pourtour de la mer Rouge et de la Corne de l'Afrique. La proximité des rives et l'ancrage historique des sociétés ont favorisé le développement d'échanges de marchandises et de populations d'une rive à l'autre sur le temps long. Le XXI ${ }^{\mathrm{e}}$ siècle s'ouvre sur la figure du réfugié yéménite quand le XIX et 
le $\mathrm{XX}^{\mathrm{e}}$ siècle ont vu prospérer celle du marchand dans les villes portuaires de la Corne de l'Afrique et de la mer Rouge (Pétriat, 2016; Miran, 2009). Ainsi, l'histoire de nombreuses familles yéménites s'est écrite au $\mathrm{XX}^{\mathrm{e}}$ siècle sur les rives arabes et africaines (De Regt, 2017). Au début $\mathrm{du} \mathrm{XX}^{\mathrm{e}}$ siècle, des Yéménites ont fui le règne despotique de l'Imamat Yahya et ont trouvé refuge à l'étranger. Des communautés yéménites, principalement issues de la plaine de la Tihama (plaine littorale occidentale du Yémen) et de la région de Taïz, ont vu le jour dans les villes portuaires d'Assab (colonie italienne d'Érythrée) ou de Djibouti Ville (colonie de la Côte française des Somalis) alors en plein développement. Il est important de rappeler ces migrations pour saisir la formation d'un capital social sur plusieurs générations. C'est sa réactualisation par les réfugiés yéménites au XXI ${ }^{e}$ siècle qui leur a permis de s'insérer socialement dans les grandes villes de la rive africaine de la mer Rouge et du golfe d'Aden.

19 Ces migrations inscrivent les parcours d'exil contemporaines des populations yéménites dans une histoire pluri-générationnelle. L'espace maritime de la mer Rouge et des eaux du golfe d'Aden a joué un rôle de soupape de sécurité. Il s'est transformé en espace de protection pour des populations désirant fuir les aléas politiques tels que l'insécurité, les formes de contrôle ou de répression.

20 L'histoire du développement des communautés yéménites à Assab et Djibouti Ville ne s'est pas conclue de la même façon. La guerre d'indépendance de l'Érythrée face à l'Éthiopie (1961 - 1993) et l'avènement du DERG, le régime autoritaire éthiopien (1974 1987), ont entraîné le déclin de la communauté yéménite d'Assab. Les familles, souvent devenues métisses arabo-éthiopiennes, sont rentrées au Yémen pour fuir l'insécurité politique. À l'inverse, la communauté arabe de Djibouti Ville a maintenu sa présence par-delà l'indépendance du pays en 1977 (Rouaud, 1997). Ainsi, en arrivant à Djibouti, certains réfugiés disposaient déjà de connaissances et de liens familiaux ou sociaux dans la capitale ou dans certaines villes de l'arrière-pays comme Obock ou Dikhil.

21 L'exil est en lui-même facteur d'inégalité parmi les réfugiés. Dans l'exil, le recours au capital social est une ressource inégalement accessible. Il existe ainsi une différence entre les réfugiés yéménites qui ont pu s'installer dans la capitale djiboutienne et ceux qui ont été contraints de s'installer au camp de réfugiés de Markazi. Les réfugiés urbains, originaires de la région de Taïz, et dont la famille dispose de contacts diasporiques à Djibouti Ville, ont pu intégrer la diaspora yéménite. Ces réseaux sociaux leur ont permis d'éviter le camp de réfugiés et d'améliorer leurs conditions d'existence via une insertion professionnelle dans les commerces et les entreprises des familles yéménites présentes depuis l'indépendance. Les conditions de vie parfois dégradées des réfugiés urbains à Djibouti Ville reflètent certes l'exode, mais elles s'inscrivent dans un projet économique de vie : les réfugiés travaillent, gagnent de l'argent, en renvoient à leur famille, se projettent pour un mariage, etc.

22 Les potentiels de survie sont aussi inégalitaires au sein du camp de réfugiés de Markazi. Le camp est ouvert en avril 2015, à quatre kilomètres de la ville d'Obock. Dès son ouverture, il accueille des familles de réfugiés venant de toutes les régions du Yémen. Les profils des réfugiés sont très hétérogènes, géographiquement, socialement et économiquement. Selon les ressources et les capacités de chacun, les situations au sein du camp évoluent différemment.

23 Le recours à la mémoire familiale est, là-encore, facteur d'adaptation dans l'exil yéménite. Parmi les communautés du camp, celle des muwalladin (les populations 
métisses yéménito-éthiopiennes) s'avère être l'une des plus soudées. Ces familles sont les descendantes des populations yéménites qui ont migré au début du $\mathrm{XX}^{\mathrm{e}}$ siècle vers la colonie italienne et la cité portuaire d'Assab. Ces familles ont prospéré pendant plusieurs décennies à Assab. Elles ont tissé des réseaux d'entraides, socio-économiques ou familiaux, au fil des générations. De nombreux yéménites se sont mariés sur la rive africaine dans des familles éthiopiennes. Avec l'avènement du DERG, ces familles sont rentrées au Yémen où elles ont connu un déclassement du fait de leur métissage : elles ont été assignées à des statuts marginalisés au sein de la société yéménite comme celui des akhdam (serviteurs) ou des muwalladin (Peutz, 2019).

$\mathrm{Au}$ Yémen, ces populations sont discriminées, notamment dans l'accès à des emplois décents. Elles ne disposent ni d'un lignage ni d'un ancrage tribal, et donc territorial. Avec la guerre, ces populations se retrouvent dans l'incapacité de se replier dans les villages ancestraux, comme de nombreux déplacés yéménites ayant quitté les grandes villes. Elles sont alors contraintes à l'exil. De fait, par leur situation commune, à Assab, puis au Yémen, et enfin à Djibouti, ces populations maintiennent des liens d'entraides importants sur plusieurs générations.

De nombreuses familles muwalladin se sont retrouvées parmi les réfugiés du camp. Les liens d'entraides du présent sont expliqués par ceux du passé : le soutien de leurs grands-parents à Assab, celle de leurs parents au Yémen, justifient la solidarité au camp de réfugiés Markazi d'Obock. L'exil au camp apparait comme un troisième temps de renforcement de ces liens de l'entraide. Cette dernière se matérialise par le partage des ressources économiques, des biens de consommation, des avantages au sein des commerces muwalladin, une solidarité accrue lors des situations d'urgence (médicale, scolaire, déplacement vers Djibouti Ville, etc.). Toutes ces manifestations de l'entraide entre les populations métisses renforcent leur communauté. Le discours de ces réfugiés s'inscrit directement dans la mémoire de leurs familles. C'est par l'évocation du passé générationnel que la communauté muwallad construit son présent et renforce son capital social.

Les réfugiés yéménites à Djibouti offrent donc à repenser les logiques de survie par l'exil et le franchissement de la frontière. L'exil est bien une perte, mais le recours à la mémoire permet soit l'arrimage à des communautés déjà existantes soit le renforcement d'une communauté malgré les aléas. Dans le cas de certains réfugiés yéménites, la frontière est un espace autant réel que symbolique qui offre l'occasion de réactualiser le capital social.

Son franchissement nécessite cependant souvent des acteurs-tiers : un ou plusieurs passeurs. Réfugiés, migrants et passeurs s'inscrivent dans un même espace frontalier. Ils forment un nœud qui se décrit par une même économie de la frontière : le franchissement de la frontière est autant un effet d'aubaine pour les migrants ou les réfugiés que pour les passeurs, les uns fonctionnant en accord avec les autres. Pour le passeur, la frontière est l'occasion de tirer profit d'une rente géographique de situation.

\section{La figure du passeur djiboutien ou la logique de l'opportunité économique de la frontière}

La deuxième figure transfrontalière étudiée est celle du passeur djiboutien. Elle donne à penser le franchissement de la frontière à l'aune d'une activité illicite : le transport de 
migrants irréguliers. Nous décrivons d'abord la route migratoire et le franchissement des frontières à Djibouti pour les passeurs et leurs convois de migrants éthiopiens. Le passage traduit ensuite la mise en connexion des territoires et des passeurs : c'est la complémentarité des échelles locales et transnationales qui permet l'existence du réseau de passeurs de l'Éthiopie à l'Arabie saoudite. Enfin, le passeur comme acteur transfrontalier est produit par un double processus économique : la sienne et celle du migrant.

Depuis le début des années 2000, le Nord de Djibouti est devenu une des routes migratoires les plus parcourues d'Afrique. Chaque année, entre 100000 et 200000 Éthiopiens quittent leur pays vers Djibouti ${ }^{4}$. Ils traversent le Nord de Djibouti et le détroit de Bab el Mandeb pour trouver du travail au Yémen, ou en Arabie saoudite depuis le début de la guerre civile yéménite. Entre l'Éthiopie et l'Arabie saoudite, les migrants doivent traverser certains États, sans connaitre au préalable la géographie des territoires et des frontières à franchir.

30 Les territoires et les lieux gardent la mémoire du vécu sociétal (Brunet, 2017 [2001]). Aussi, cette route migratoire n'est pas nouvelle en soi. Quatre temps historiques se superposent sur ce parcours. Jusqu'au début du $\mathrm{XX}^{\mathrm{e}}$ siècle, cette route fait partie d'un système économique esclavagiste régional reliant l'Abyssinie aux royaumes de la Péninsule arabique. Ce système s'essouffle dans la première moitié du $\mathrm{XX}^{\mathrm{e}}$ siècle. La route passant par Tadjoura et le Nord de Djibouti est alors la dernière à se tarir (Dubois, 2013). L'esclavage n'est plus mais la migration demeure. La route est utilisée par des passeurs: des bergers, des pêcheurs, des habitants de la région qui font transiter quelques centaines ou milliers de migrants vers les villes yéménites, demandeuses en main-d'œuvre. Durant la deuxième moitié du $\mathrm{XX}^{\mathrm{e}}$ siècle, la population yéménite masculine émigre en effet massivement vers le royaume saoudien alors en plein boom pétrolier, créant un appel d'air pour la main-d'œuvre du pays (Thiollet, 2004). Les travailleurs éthiopiens remplacent cette main-d'œuvre yéménite partie en Arabie saoudite, que la précarité des conditions rend volatile (De Regt, 2012). Enfin, la première décennie du XXI siècle s'ouvre sur une migration massive des Éthiopiens vers la Péninsule arabique. Les migrants quittent l'Éthiopie vers Djibouti pour traverser le détroit de Bab el Mandeb. Certains migrants, parmi les plus précaires, tentent de traverser ces étendues seuls. D'autres font appel à un réseau de passeurs couvrant le parcours migratoire.

31 Le franchissement de la frontière par les passeurs djiboutiens et les convois de migrants éthiopiens est simple à décrire. La frontière entre l'Éthiopie et Djibouti sépare une étendue désertique continue. Une unique route relie la capitale, Djibouti Ville, à l'Éthiopie, dans l'ouest djiboutien. Seul le poste des douanes de Gâlâfi symbolise l'institution étatique entre les deux États. Cette frontière ouest de Djibouti est emblématique des confins isolés, peu surveillés, dans lesquels la faible présence étatique ouvre des possibilités de trafic et de contrebande (Bennafla, 2014).

32 À l'est, le détroit de Bab el Mandeb, à la jonction des eaux de la mer Rouge et du golfe d'Aden, sépare Djibouti et le Yémen. Là encore, malgré la présence des garde-côtes dans le village côtier de Khor Angar, les faibles capacités de surveillance de l'État djiboutien et l'importance des plages et criques désertes permettent aux passeurs djiboutiens et aux dalals (passeurs) yéménites ${ }^{5}$ de se rencontrer et de charger les migrants sur des embarcations arabes. Les migrants sont transportés en véhicule, de nuit, de relais en relais, d'Éthiopie jusqu'aux plages à l'extrême nord de Djibouti. 

mobilité géographique perçue comme condition de mobilité sociale et par la formation transnationale d'un réseau de passeurs. Ce réseau regroupe des passeurs éthiopiens, djiboutiens, yéménites et saoudiens connectés entre eux. Ils forment une chaîne coordonnée, faisant transiter les migrants éthiopiens de relais en relais, d'un État à l'autre. La mise en connexion de ces acteurs permet une meilleure logistique des territoires et des convois. La fréquence et la quantité de migrants transportables est donc revue à la hausse. Ce réseau de passeurs articule les échelles locales et transnationales. La situation relève alors du paradoxe des frontières traversées de flux clandestins. Le passeur naît du besoin de faire franchir des frontières nationales à un groupe de populations. Pourtant, par leur action, les passeurs illustrent une négation des frontières et du territoire national de chaque État sur cette route migratoire.

du passeur s'appuie sur les limites du territoire national et du contrôle de l'État aux frontières. Il est un acteur dont l'existence est induite directement par la difficulté de franchir la frontière pour une population migrante. Cette difficulté est physique (la mer) aussi bien que liée au manque de connaissance du territoire à traverser. Le passeur fait ainsi traverser la frontière (une ligne), le territoire (une surface) en garantissant une protection à sa "marchandise", notamment lors des situations complexes (les contrôles des forces régaliennes ou la traversée de la mer, etc.). L'action de ce dernier est d'autant plus forte qu'elle s'inscrit dans les interstices des États et qu'elle illustre précisément la faiblesse étatique à contrôler son territoire. Faire transiter des migrants s'inscrit dans un couple local - transnational faisant fi du national. Le passeur tire son activité d'une demande de franchissement de la frontière et des connaissances du territoire. Dans le cadre du réseau transnational des passeurs, c'est la somme des connaissances géographiques et sociales et du pouvoir économique 
des passeurs, à l'échelle locale, qui permet le fonctionnement du réseau, de relais en relais.

Le passage de frontière est un effet d'aubaine autant pour celui qui la traverse que pour le passeur qui la fait traverser. Le passeur naît d'une demande de mouvement, celle du migrant. Il répond alors à un premier besoin économique : celui d'une population de migrer ou de fuir un contexte donné. La migration qu'entreprennent les Éthiopiens vers l'Arabie saoudite s'apparente à une migration économique. Ils souhaitent travailler au Yémen ou en Arabie saoudite pour y amasser un capital. Ils y travaillent plusieurs années avant de rentrer - expulsés ou volontairement - en Éthiopie. L'Arabie saoudite comme le Liban sont des destinations qui permettent cette mobilité sociale. L'objectif est de partir pour mieux rester : le retour en Éthiopie est l'occasion de développer une activité économique ou la construction d'un foyer, matérialisant ainsi la réussite économique de la migration (Chiré et Tamru, 2016).

Devenir passeur correspond également à une logique de survie économique qui permet de subvenir à ses besoins ou ceux de sa famille. Le passeur est souvent jeune, sans perspective d'avenir identifiée et issu de familles précaires. Il ne dispose pas d'un capital social suffisant pour lui permettre d'intégrer économiquement Djibouti Ville. Son capital social s'inscrit à l'échelle locale, dans une région politiquement marginalisée par l'État djiboutien. Le trafic de migrants lui permet alors de développer son propre capital socio-économique en exploitant une rente géographique de situation (Magrin, 2013).

Le passeur définit un prix à payer pour le migrant qui souhaite traverser la frontière. Un migrant éthiopien paye l'équivalent d'une somme comprise entre 1000 et 2000 euros de l'Éthiopie jusqu'en Arabie saoudite. Les différents passeurs et relais la partagent ensuite. Ce prix que le migrant paye correspond à deux éléments. Il symbolise d'abord le prix du passage. Le migrant paye pour un service qui consiste à franchir des frontières, être transporté d'un point $\mathrm{A}$ à un point $\mathrm{B}$. Être passeur est donc une activité économique légitime autant pour le migrant que pour le passeur qui en tire profit. Le prix à payer comprend ensuite le droit de passer sur un territoire donné : en franchissant illégalement la frontière, le migrant verse dans l'illégalité. En situation irrégulière, il n'a pas de visa ou d'autorisation de l'administration djiboutienne pour justifier sa présence. Il peut donc être, théoriquement, expulsé du territoire djiboutien : c'est notamment le cas d'un nombre important de migrants éthiopiens à Djibouti Ville, dont la faiblesse de l'ancrage en milieu urbain permet de fréquentes reconduites à la frontière (Chiré, 2018). L'intégration régionale des États de la Corne de l'Afrique n'inclut pas la libre circulation de la population au sein des États membre. L'organisation des passeurs singe l'organisation étatique.

40 Le passeur djiboutien répond donc à une double crise. Il est généré par la crise interne éthiopienne. Les migrants éthiopiens décident de partir tenter leur chance en Arabie saoudite car ils estiment n'avoir aucune possibilité économique en Éthiopie. Les passeurs inscrivent ensuite leur activité dans une justification politique, en contestant leur marginalisation par l'État djiboutien. Le Nord de Djibouti est une région délaissée où les éventualités économiques demeurent très faibles. Le détour par l'illicite se justifie par l'illégitimité de l'État : les passeurs reprennent ainsi l'argumentaire défendu par les rebelles afars du FRUD dès 1991 pour légitimer leur activité (cf. partie suivante). De fait, l'activité du passeur représente une négation autant de la justice djiboutienne 
(qui condamne le trafic) que de la souveraineté étatique aux frontières, faisant évoluer la relation que les passeurs ont de l'espace qui les entoure.

41 Le passeur djiboutien permet la mise en connexion (illicite) des territoires et des populations. Il esquive l'échelle nationale pour valoriser le couple local-transnational. La somme de ses connaissances à l'échelle locale permet, de relais en relais, de créer des parcours transnationaux. L'activité du passeur est une forme de réponse économique à la politique de marginalisation imposée par l'État au Nord de Djibouti depuis plusieurs décennies. Elle s'apparente à une résistance passive face à l'État djiboutien et s'inscrit dans la continuité d'une histoire mouvementée entre cette région du Nord de Djibouti et le gouvernement djiboutien. Deux décennies avant l'avènement de ces nouveaux passeurs, la région était l'épicentre de la guerre civile djiboutienne. La résistance n'était pas passive mais armée, et incarnée par la figure du rebelle afar.

\section{La figure du rebelle afar ou la logique de soutien là où la souveraineté de l'État s'arrête}

La troisième figure transfrontalière constitutive de l'espace d'étude est celle du rebelle afar. Elle illustre les articulations entre lutte armée et passage de frontière. Cette partie présente d'abord, comme éléments de contexte, la guerre civile djiboutienne de 1991 à 1994. Ensuite est expliquée l'importance du franchissement de la frontière dans la guerre civile comme logique de soutien aux deux belligérants. Enfin, nous analysons le rebelle comme acteur transfrontalier, en montrant comment l'international est une condition du maintien de la lutte.

La guerre civile djiboutienne a opposé la rébellion du Front pour la Restauration de l'Unité et de la Démocratie (FRUD) à l'État djiboutien de 1991 à 1994. Le FRUD a trouvé son origine dans les revendications d'une partie de la population afar du Nord de Djibouti pour un meilleur partage du pouvoir politique entre les Afars et les IssasSomalis, qui forment l'ossature du gouvernement djiboutien. La marginalisation des Afars depuis l'indépendance de 1977 a conduit à un fort sentiment d'injustice chez ces populations (Mohamed, 2002). En 1991, le FRUD a pris les armes.

En quelques semaines, le FRUD a conquis les deux-tiers du territoire djiboutien et s'est arrêté aux portes de la capitale. L'armée française est intervenue, à la demande du président Gouled, pour maintenir le gouvernement et entamer des pourparlers. Des négociations ont été entreprises mais ces dernières ont été ponctuées par des massacres de population (Djibouti Ville, quartier d'Arhiba le 18 décembre 1991), des escarmouches entre rebelles et forces régulières dans le Nord du pays, notamment pour la tentative de la prise de la ville d'Obock par les rebelles. En 1994, des accords de paix sont signés, censés aboutir à un partage des pouvoirs, à travers notamment un processus de décentralisation. L'objectif était de permettre aux régions afars du Nord de Djibouti de s'auto-administrer. Dans un État comme Djibouti, où la capitale concentre près de 70 à $80 \%$ de la population (Chiré, 2012), la décentralisation devait permettre aux régions Nord d'obtenir une certaine autonomie. Or cette clause de l'armistice ne fut jamais effective. La guerre se solda par l'intégration des chefs rebelles au sein du gouvernement djiboutien via l'obtention de postes dans certains ministères. De fait, cette solution ne trouva pas un large écho au sein de la population afar. Si le FRUD a intégré le jeu des partis politiques, une branche s'en est séparée pour reprendre la lutte armée, dans le maquis, jusqu'en 2001, date d'un nouvel accord de paix. Une 
troisième branche du FRUD s'est ensuite constituée pour continuer la lutte armée. Les derniers rebelles sont installés dans l'extrême nord du territoire djiboutien, dans la zone frontalière avec les régions afars d'Éthiopie et d'Érythrée.

Illustration 2 - Zones linguistiques et frontières autour de Djibouti

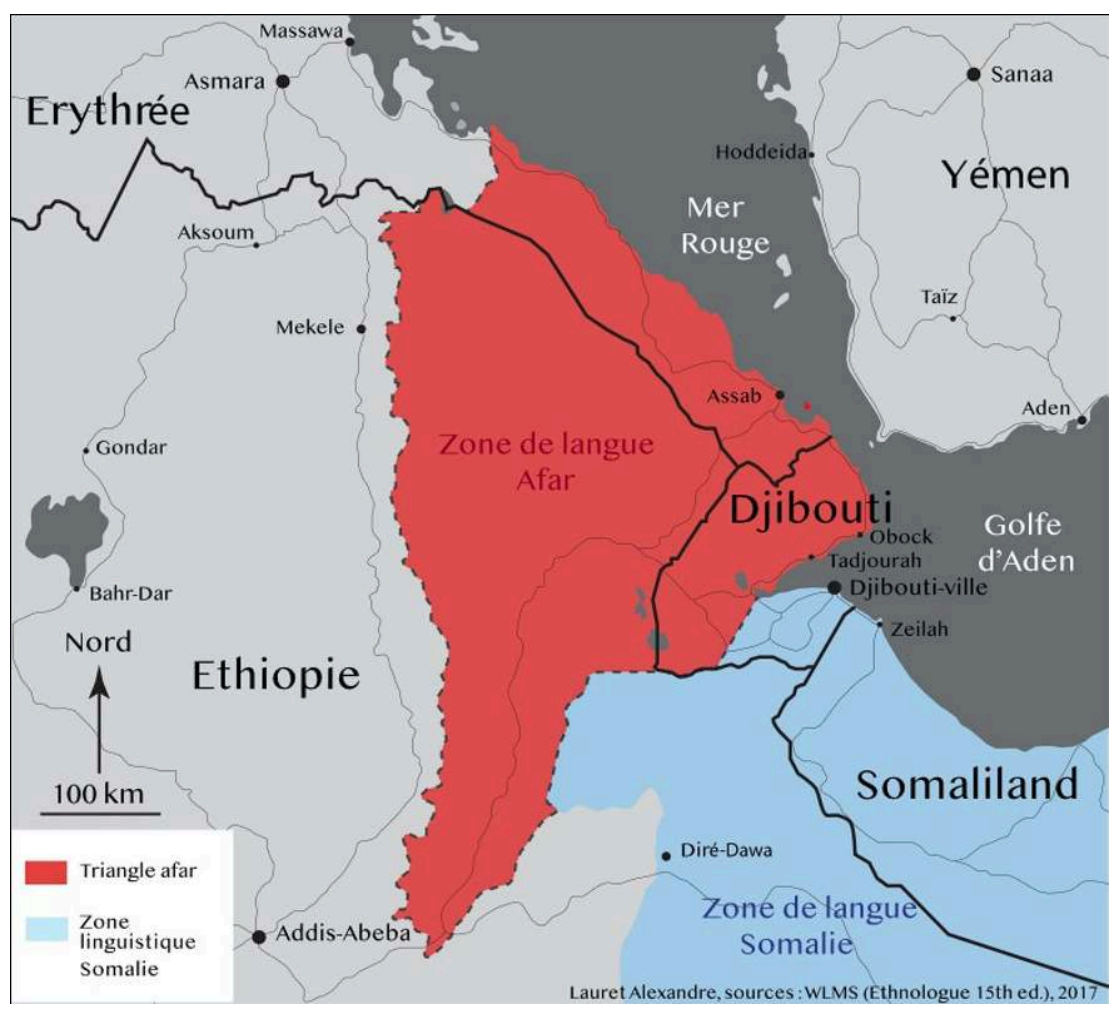

Source: World Language Mapping System (WLMS), 2017. Auteur: A. Lauret

Dès le début de la guerre civile, un glissement de la grille de lecture du conflit est apparu: ce n'était plus la rébellion des Afars de Djibouti contre le gouvernement djiboutien, mais une guerre des Afars contre les Somalis. Le « triangle » afar se compose en effet de trois régions limitrophes : en Éthiopie, en Érythrée et à Djibouti. Les rebelles étaient d'abord des Afars djiboutiens. Mais très vite, le FRUD a bénéficié d'un large soutien au sein des différentes régions afars: des combattants afars d'Éthiopie et d'Érythrée sont venus grossir les rangs de la rébellion.

Cet appui constant dont les rebelles ont bénéficié depuis la guerre civile éclaire la nature de la figure du rebelle. Ce dernier reçoit du soutien depuis l'étranger et passe la frontière pour obtenir du soutien. La lutte se déploie non plus uniquement à l'échelle nationale, mais à l'échelle transnationale. La guerre civile djiboutienne a été nourrie par plusieurs phénomènes transfrontaliers. D'abord, les régions afars d'Éthiopie et d'Érythrée ont fourni un réservoir de combattants et d'armes (Le Gouriellec, 2020) : de nombreux Afars de ces régions avaient pris part à des combats contre le DERG dans les années 1980 et dans la guerre d'indépendance d'Érythrée. Beaucoup étaient déjà équipés en armes issues de ces affrontements ou récupérées au Yémen. La proximité des rives et des populations a permis d'acheminer des armes directement du Yémen vers le Nord de Djibouti ou en passant par l'Érythrée. Les intermédiaires sur les deux rives étaient ou bien des rebelles dissimulés sur les différents territoires, ou bien des 
intermédiaires locaux, avec qui les rebelles avaient entretenu des rapports sociaux du fait de la proximité des familles, des lignages ou des alliances économiques.

Par ailleurs, la guerre civile djiboutienne s'illustre autant comme une guerre civile nationale que comme le réceptacle des rivalités ethniques et politiques à l'échelle de la Corne de l'Afrique. Si la rébellion du FRUD a été soutenue par les régions afars limitrophes, le gouvernement djiboutien a aussi reçu de l'aide extérieure. Ce dernier, dont l'armée avait été prise de court en 1991, a intégré massivement des Somalis-Issas d'Éthiopie et du Somaliland au sein de l'armée et des services pour appuyer les combats (Bezabeh, 2011). Ces campagnes de recrutement de soldats à l'étranger ont renforcé l'argumentaire ethnique au sein des enjeux politiques à Djibouti. Le recrutement d'éléments étrangers autant afars que somalis donne à penser la frontière comme un élément peu déterminant dans le conflit national djiboutien.

Enfin, le maintien de la rébellion depuis 2001 s'explique par le territoire dans lequel les rebelles survivent: l'extrême nord de Djibouti. C'est une région frontalière montagneuse, difficile d'accès, où la présence étatique demeure très faible. Il existe des liens entre les rebelles afars djiboutiens et les populations des régions limitrophes. La fermeture de la frontière entre Djibouti et l'Érythrée depuis l'incident de Douméra ${ }^{6}$ en 2008 est paradoxale. La fermeture n'est effective que sur quelques kilomètres de frontière officielle contrôlés par le gouvernement, puisque les rebelles y sont positionnés sur une autre partie importante.

Un double jeu de la frontière apparait. Pour les rebelles afars, la frontière entre les trois territoires afars s'est effacée. Bien qu'il existe des différences entre eux, les Afars estiment qu'ils ont le droit de se déplacer librement au sein des territoires du triangle afar. Cet effacement légitime l'entrée de combattants non djiboutiens et le franchissement de la frontière pour recevoir de l'aide. La frontière étatique est donc peu ou faiblement reconnue. Or, pour l'État djiboutien, son action au-delà de son territoire - et donc de sa frontière - n'est pas possible sans déclencher des représailles de l'Érythrée. Ce sont donc deux visions du territoire et de la souveraineté qui s'affrontent, au-delà de ces escarmouches. Or les actions répressives de l'État ne peuvent s'inscrire que dans le cadre national, ce qui n'est pas le cas du rebelle : là réside sa logique de survie.

Le rebelle afar est un acteur transfrontalier, dont le maintien de la lutte armée suppose la possibilité de franchir la frontière. Dans le cadre de la guerre civile djiboutienne, les régions afars des États adjacents ont représenté des territoires sur lesquels la rébellion du FRUD s'est appuyée pour se maintenir, en 1991, puis en 1994, enfin depuis 2001.

Le franchissement de la frontière par le rebelle est apparu dès le commencement du conflit comme une ressource permettant d'améliorer ses chances de lutter. La rive nord du golfe de Tadjoura fut le théatre d'affrontements entre rebelles et forces loyalistes. La ville d'Obock est devenue l'épicentre du conflit. Elle fut touchée par les combats et les destructions. Les populations décidèrent alors de fuir dans les familles, proches ou lointaines, à l'étranger, en Érythrée ou au Yémen. Ce franchissement des frontières ressemble à celui des réfugiés yéménites lors de la guerre civile de 2014. Pour les combattants et les rebelles, qui seront défaits à Obock, le passage de la frontière représente un espace refuge et de sécurité sur lequel l'État djiboutien ne peut se projeter. Il rend possible le glissement d'une lutte nationale vers une lutte depuis l'étranger. Les moyens d'actions évoluent : la lutte depuis l'étranger pousse les rebelles à utiliser des canaux d'actions différents comme la médiatisation de la lutte ou 
l'utilisation de discours à la place des armes. Les nouvelles technologies permettent de médiatiser la lutte et offrent des nouveaux espaces de dialogue et de négociation pour les rebelles. L'étranger devient une ressource : en France ou en Europe, les opérations médiatiques des réfugiés politiques s'organisent devant des administrations ou des ambassades.

Franchir la frontière est un moyen de résister. C'est un espace refuge d'autant plus efficace que l'État djiboutien voit sa souveraineté limitée. Le Yémen a offert aux rebelles afars, dès la guerre civile, la possibilité d'une base arrière pendant toute la durée du conflit. Les armes que la rébellion utilisaient venaient tantôt d'Éthiopie ou d'Érythrée, tantôt de soutiens au Yémen. Les rebelles afars en exil y ont pu trouver des vivres, des armes et un soutien logistique permettant de maintenir l'effort de guerre. Face à cette situation, le gouvernement djiboutien demanda l'extradition de certains combattants et leaders au gouvernement yéménite. Ces demandes furent sans succès : les rebelles avaient noué des attaches historiques, pluri-générationnelles, avec certaines populations de la côte yéménite. Ils étaient invités à séjourner au Yémen. Quant au gouvernement yéménite, il préféra adopter un rôle de médiateur dans les processus de paix.

\section{Conclusion : vers une nouvelle géographie du Sud de la mer Rouge}

53 À travers les figures du réfugié yéménite, du passeur djiboutien et du rebelle afar, cet article montre que les crises favorisent l'émergence de nouveaux espaces transfrontaliers. Ces derniers sont matérialisés par le franchissement de la frontière par chacun de ces acteurs. Les logiques et les méthodes pour passer la frontière diffèrent, mais la traversée répond au même enjeu: mobiliser des ressources essentielles.

54 L'instabilité de la Corne de l'Afrique et du Yémen, matérialisée par la récurrence des crises, engendre donc des acteurs transfrontaliers. Le passage de la frontière est ici un moyen de gérer les aléas politiques. L'exil des réfugiés yéménites permet la survie du corps et s'accompagne du recours à des mécanismes d'entraide transgénérationnelles ou multi-situées. L'activité du passeur djiboutien illustre une double logique d'oppression et d'opportunité : la nécessité pousse le migrant éthiopien à rechercher à l'étranger des ressources nouvelles; le passeur quant à lui recherche des occasions d'enrichissement qui répondent à l'absence de l'État djiboutien. Enfin, pour le rebelle afar, la frontière est autant un espace ressource sur lequel s'appuyer pour mener son combat qu'un espace refuge pour s'exiler lors des revers de la lutte.

Les crises ouvrent de nouveaux espaces d'échanges face à des États qui s'enlisent: effondrement, morcellement, fermeture des frontières, révolution et troubles internes. Les populations étudiées ici s'affranchissent des frontières pour évoluer en dehors des cadres étatiques définis. Une nouvelle géographie est rendue possible par les logiques de survie des acteurs transfrontaliers qui agissent sur les territoires. Si les frontières restent le symbole de la souveraineté étatique, cette souveraineté est contestée par les franchissements perpétuels. La concentration des études sur les États fait que de nombreuses analyses sur la région sont aveugles aux phénomènes transfrontaliers : que représente la fermeture de la frontière entre Djibouti et l'Érythrée, décidée par les deux 
États en guerre, alors que les populations afars d'Obock et de Raheita sont libres de circuler en dehors d'un unique check-point officiellement contrôlé ? Il en est de même pour les réfugiés yéménites d'Obock, dont une partie fait des aller-retours entre le camp de réfugiés et le Yémen, au gré des péripéties de la guerre et des profits économiques possibles. Le détour par les crises et par les acteurs transfrontaliers montre une géographie alternative, vivante et en constante évolution de la Corne de l'Afrique et du Yémen, quand bien même les frontières semblent fermées sur la carte.

\section{BIBLIOGRAPHIE}

Adnan E., 2020. Voyage, guerre, exil. Paris, L'échoppe, 45 p.

Bennafla K., Peraldi M., 2008. Introduction. Frontières et logiques de passage : l'ordinaire des transgressions. Cultures et conflits [En ligne], nº 72, p. 7-12. URL: http://journals.openedition.org/ conflits/17383 - DOI: https://doi.org/10.4000/conflits.17383

Bennafla K., 2014. État et illégalisme : Quelle géographie ? Une approche par les flux marchands depuis l'Afrique et le Moyen Orient. Annales de géographie, n 700, p. 1338-1358.

Bezabeh S. A., 2011. Citizenship and the logic of sovereignty in Djibouti. African Affairs, p. 1-20.

Bozzini D., 2014. Surveillance, répression et construction collective de l'insécurité en Erythrée. Politique africaine, $\mathrm{n}^{\circ} 135$, p. 137-157.

Brunet R., 2017 [2001]. Le déchiffrement du monde : Théorie et pratique de la géographie. Paris, Belin, $528 \mathrm{p}$.

Canguilhem G., 2000 [1952]. La connaissance de la vie. Paris, Librairie philosophique Vrin, 198 p.

Chiré A. S., 2012. Djibouti contemporain. Karthala, 380 p.

Chiré A. S., Tamru B., 2016. Les migrantes de retour dans la Corne de l'Afrique : vers une transformation sociale des espaces émetteurs, le cas éthiopien. Echogéo [En ligne], $\mathrm{n}^{\circ}$ 37. URL: http://journals.openedition.org/echogeo/14708 - DOI : https://doi.org/10.4000/echogeo.14708

Chiré A. S., 2018. De l'insertion urbain à l'administration plurielle des migrants régionaux dans l'agglomération djiboutienne. Annales d'Éthiopie, nº 32, p. 67-94.

De Regt M., 2012. Ethiopian Women's Migration to Yemen: past and present. Arabian humanities [En ligne], n 17, p. 1-15. URL: http://journals.openedition.org/cy/1853 - DOI: https://doi.org/ 10.4000/cy.1853

De Regt M., 2017. From Yemen to Eritrea and back: A Twentieh Century Family History. Northeast African Studies, vol. 17, n 1, p. 26-49.

Dubois C., 2013. Une traite tardive en mer Rouge méridionale : la route des esclaves du golfe de Tadjoura (1880 - 1936). In Médard H., Derat M.-L., Vernet T., Ballarin M.-P. (ed.), Traites et esclavagistes en Afrique orientale dans l'Océan Indien. Paris, Karthala, p. 197-222.

Feyissa D., Hoehne M. V., 2010. Borders \& borderlands as resources in the Horn of Africa. Martlesham, James Currey Edition, $224 \mathrm{p}$. 
Gascon A., 1990. La guerre comme rite géographique : l'exemple de la Corne de l'Afrique. Cultures et conflits [En ligne], $\mathrm{n}^{\circ}$ 1, p. 69-84. URL: http://journals.openedition.org/conflits/78 - DOI: https://doi.org/10.4000/conflits.78

Le Gouriellec S., 2020. Djibouti : la diplomatie de géant d'un petit État. Villeneuve d'Ascq, Septentrion Presses universitaires, $224 \mathrm{p}$.

Magrin G., 2013. Voyage en Afrique rentière : une lecture géographique des trajectoires du développement. Paris, Publications de la Sorbonne, 424 p.

Mermier F., 2018. Yémen : Écrire la guerre. Paris, Classique Garnier, 192 p.

Mohamed A., 2002. Ourrou Djibouti 1991 - 1994 : du maquis afar à la paix des braves. Paris, L'Harmattan, 266 p.

Miran J., 2009. Red Sea Citizens: Cosmopolitan society and cultural change in Massawa. Indiana University Press, $400 \mathrm{p}$.

Pétriat P., 2016. Le négoce des lieux saints : négociants hadramis de Djedda 1850 - 1950. Paris, Publications de la Sorbonne, $464 \mathrm{p}$.

Peutz N., 2019. “The fault of our grandfathers": Yemen's third-generation migrants seeking refuge from displacement. Journal of Middle East Studies [En ligne], n 51, p. 357-376. DOI: https:// doi.org/10.1017/S0020743819000370

Raeymaekers T., 2011. African boundaries and the new capitalist frontier. In Wilson T. M., Donnan H., A companion to boerder studies. Wiley-Blackwell, p. 318-331.

Rouaud A., 1997. Pour une histoire des Arabes à Djibouti (1896 - 1977). Cahier d'études africaines, vol. 37, n 146, p. 319-348.

Thiollet H., 2004. Aux marges du monde arabe : Place du Yémen dans les itinéraires de migrants et de réfugiés érythréens. Arabian Humanities [En ligne]. URL: http://journals.openedition.org/cy/ 190 - DOI: https://doi.org/10.4000/cy.190

\section{NOTES}

1. Yemen Emergency - UNHCR https://www.unhcr.org/yemen-emergency.html [Consulté le 1 septembre 2020].

2. On peut rappeler ici la guerre civile de 1994 entre le Nord et le Sud, puis les six guerres de Saada entre le gouvernement yéménite et les Houthis durant la décennie 2000, enfin la révolution de 2011 qui emporta le gouvernement de A. Saleh.

3. Les Yéménites qui arrivent à Djibouti obtiennent le statut de réfugié selon le prima facie.

4. La donnée est issue d'entretiens avec des responsables des Nations Unies et de l'Organisation Internationale pour les Migrations (OIM) à Djibouti (juin et juillet 2019).

5. Le terme de dalal signifie avant tout un courtier ou un intermédiaire commercial. C'est un terme que l'on retrouve autant dans la sphère économique des passeurs que dans le commerce de bétail et dans certains contextes, de manière plus large, comme l'achat d'un terrain ou d'une maison.

6. Une escarmouche a lieu entre les armées djiboutiennes et érythréennes en juin 2008, à la suite de revendications communes du point frontalier de Douméra, donnant sur le détroit de Bab el Mandeb. 


\section{RÉSUMÉS}

La Corne de l'Afrique et le Yémen représentent une région en proie à de multiples crises politiques et territoriales. Cet article souhaite comprendre comment ces crises favorisent de nouveaux espaces d'échanges à trois types d'acteurs différents : le réfugié yéménite fuyant la guerre, le passeur djiboutien faisant transiter des migrants éthiopiens et le rebelle afar en lutte contre l'État djiboutien. Ces trois figures sont générées par les crises et le franchissement de la frontière. Elles illustrent chacune une logique de survie différente: franchir la frontière pour l'exil, l'opportunité économique, enfin le soutien d'une lutte armée depuis l'étranger. Ces situations donnent à réfléchir aux stratégies que les populations mettent en place pour gérer les aléas politiques des États de la Corne et du Yémen.

The Horn of Africa and Yemen represent an area subject to numerous political crises and territorial disputes. The present article aims to comprehend how the crises foster new places of exchange between three different types of stakeholders: the Yemenite refugee fleeing war, the smuggler routing Ethiopian migrants, and the Afar rebel fighting the Djiboutian government. Each of them gives a specific example of a strategy for survival: crossing the border for exile, economic opportunity, and the support of an armed rebellion from abroad. Those three strategies invite reflection on how populations react to deal with local political hazards in the countries of Yemen and the Horn of Africa.

\section{INDEX}

Mots-clés : Corne de l'Afrique, Yémen, Djibouti, frontière, réfugié, passeur, rebelle Keywords : Horn of Africa, Yemen, Djibouti, border, refugee, smuggler, rebel

\section{AUTEUR}

\section{ALEXANDRE LAURET}

Alexandre Lauret, al.lauret@gmail.com, est doctorant à l'Université Paris VIII Vincennes - SaintDenis, laboratoire LADYSS. Il contribue au site Bab-el-Mandeb, carnet de recherche sur Djibouti et sa région. Il a récemment publié :

- Lauret A., 2020. Du réfugié yéménite à l'entrepreneur. Quand l'exil de guerre devient opportunité économique à Djibouti. Politique africaine, $\mathrm{n}^{\circ}$ 159, p. 145-168.

- Lauret A., 2018. Djibouti et le Yémen : influences, interdépendances et échanges des pêcheurs d'une rive à l'autre. Arabian Humanities [En ligne], $\mathrm{n}^{\circ}$ 10. URL: http://journals.openedition.org/cy/ 3798 - DOI: https://doi.org/10.4000/cy.3798 